\title{
Triangular backgrounds shift the bias of line bisection performance in hemispatial neglect
}

\author{
M B Shulman, M P Alexander, R McGlinchey-Berroth, W Milberg
}

J Neurol Neurosurg Psychiatry 2002;72:68-72

See end of article for authors' affiliations

.....................

Correspondence to: DrMP Alexander, Behavioral Neurology Unit, KS-2, Beth Israel

Deaconess Medical Center 330 Brookline Avenue, Boston, MA 02215, USA malexand@ caregroup.harvard.edu

Received

26 January 2001

In final revised form 10 August 2001

Accepted 10 September 2001

\begin{abstract}
Objective: Patients with left neglect on line bisection show normal implicit sensitivity to manipulations of both the stimulus and the visual background. Three experiments were designed to define this sensitivity more exactly.

Methods: Normal controls and patients with left neglect performed a series of horizontal line bisection tasks. Independent variables were the configurations of the backgrounds for the line-rectangle, square, circle, left and right pointing isosceles triangles-and whether the background was the shape of the piece of paper or an outline drawn on a standard piece of paper. In a separate experiment different components of the triangle were outlined on a piece of paper. Deviation from true midpoint was calculated.

Results: Simply placing the target lines in a symmetric background such as a square or circle did not reliably reduce neglect. A triangle asymmetric in the horizontal plane caused a shift in bisection away from the triangle's vertex. With right pointing triangles the perceived midpoint shifted to the left of true centre (crossed over). The effects of the triangles were comparable in the patients and the controls when controlled for baseline bisection bias. The critical components of the triangles were the angular legs. This effect of background was not influenced by lesion site or by hemianopia.

Conclusions: Patients with left visual neglect remain sensitive to covert manipulations of the visual background that implicitly shift the perceived midpoint of a horizontal line. This effect is strong enough to eliminate neglect on a bisection task. The mechanism of this effect is expressed through preattentive visual capacities.
\end{abstract}

$\mathrm{P}$ atients with hemispatial neglect after right hemispheric damage fail to report or respond explicitly to stimuli appearing in the left hemispace. Bisection of horizontal lines is commonly used at the bedside to detect neglect, and right hemispheric damage results in displacement of the mark to the ipsilateral (right) side. This phenomenon is deceptively simple, as it can be dramatically influenced by many properties of the stimulus (the length of the horizontal line), the background (the page), the visual field in which the task is presented, and the context in which the stimulus appears.

The length of the line is positively correlated with the extent of rightward bias. ${ }^{.}$Very short lines can produce a "crossover" effect in which the line is bisected left of the actual midpoint despite a right sided lesion. ${ }^{2-5}$ Whatever its length, no line stands entirely free of context. Even long lines may be bisected unexpectedly to the left ("crossover") if the immediately preceding line was longer. ${ }^{6}$ Altering context by making small changes in line morphology can produce substantial effects on neglect. There are well known perceptual illusions created by adding fins $(<-<)$ to a line's ends. Both the illusion of length (Muller-Lyer: $>-<$ appears longer than $<->$ ) and the illusion of perceived midpoint (Judd: $>->$ appears to have a midpoint to the left of the true midpoint) are present in the line bisection of patients with neglect. ${ }^{7-9}$

The page of paper on which a line is presented is generally considered a passive vehicle, but it too can exert a powerful influence on the extent of bisection displacement. ${ }^{10}$ For instance, Marshall and Halligan ${ }^{4}$ reported that a patient with severe, persistent neglect evidenced a striking reduction of bisection displacement when the line to be bisected was centred in square or circular pieces of paper. ${ }^{11}$ Regular symmetry of the background seemed to affect neglect, as bisection progressively improved as page shape went from rectangle to square to circle. This effect of background is consonant with the same investigators' previous demonstration that changing the bisection task to marking the centre of a geometric shape also influences neglect. The magnitude of midpoint displacement is inversely related to the "height" of the figure from "flat" rectangle to square or from "flat" ellipse to circle.

In all of the tasks summarised above, the conscious, attention demanding requirement is locating the midpoint of some stimulus, usually a horizontal line. All other aspects of the task that influence performance are thought to be "preattentive", that is, they utilise operations in visual perception that occur in the absence of directed attention or awareness. ${ }^{12}$

The experiments reported here were motivated by observations about preattentive processing of the context of stimulus and of background information on neglect. We had two goals. Firstly, most of the observations about the effects of background manipulation come from single case studies, often of patients with very severe, and unusually persistent, neglect. For example, the claims about background (paper) shape were made in a patient with left hemianopia in addition to neglect, who, several months after stroke, still had a mean deviation of $60 \mathrm{~mm}$ on bisection of $180 \mathrm{~mm}$ lines centred in a rectangle. It is not known if these effects are so readily demonstrated in patients with more typical and milder neglect. In the case report, ${ }^{11}$ the authors essentially request that such a confirmation be performed.

Secondly, in the studies of Halligan and Marshall, ${ }^{4}$ both "height" and "length" of the stimulus figure or the background were manipulated, but all were symmetric about both horizontal and vertical axes. Incorporation of the horizontal bias in the Judd illusion $(>->)$ into the background should markedly shift midpoint determinations in patients with neglect, just as the simple illusion does in

Abbreviations: TD, transaction displacement 


\begin{tabular}{|c|c|c|c|c|c|c|}
\hline Patient & Age & Sex & Lesion site & $\begin{array}{l}\text { Visual field } \\
\text { defect }\end{array}$ & $\begin{array}{l}\text { Weeks } \\
\text { post-injury }\end{array}$ & Hemiparesis \\
\hline 1 & 84 & $\mathrm{~F}$ & ॥ & None & 2 & + \\
\hline 2 & 60 & M & I, II, III & LHH & 8 & + \\
\hline 3 & 78 & M & I & None & 5 & + \\
\hline 4 & 85 & M & II, III & None & 4 & - \\
\hline 5 & 83 & $\mathrm{~F}$ & I, II, III & LHH & 6 & + \\
\hline 6 & 78 & $\mathrm{~F}$ & $\|$ & None & 3 & + \\
\hline 7 & 58 & $\mathrm{~F}$ & 1 & LIQ & 6 & + \\
\hline 8 & 49 & M & I, II & None & 3 & + \\
\hline 9 & 84 & $\mathrm{~F}$ & $\|$ & None & 12 & + \\
\hline 10 & 62 & M & I, II, III & None & 7 & + \\
\hline 11 & 62 & M & I, II, III & LIQ & 10 & + \\
\hline 12 & 74 & $\mathrm{~F}$ & III & None & 2 & - \\
\hline 13 & 74 & M & II, III & None & 6 & + \\
\hline 14 & 55 & $\mathrm{~F}$ & ॥ & None & 2 & + \\
\hline
\end{tabular}

normal people. Presuming that the direction of the fins shifts perceived midline, then a background of an isosceles triangle with base perpendicular to the stimulus line should have the same directional effect: neglect reduced for right apex triangles and increased for left apex triangles.

We report the findings in a larger sample of patients in three experiments that manipulated background (page) shape (experiment 1: rectangular, square, circular, left triangle, right triangle), outlined shape on paper (experiment 2: left triangle, right triangle), and the outlined shape with different aspects of the triangle eliminated (experiment 3 ).

\section{EXPERIMENT 1}

\section{Subjects}

All patients with documented right hemispheric stroke as seen on CT or MRI who were admitted to the inpatient service of an acute rehabilitation hospital were identified. Only patients who spoke English and whose primary physician thought would be able to cooperate during the testing protocol were approached to participate. Patients were excluded from participation if there was a history of stroke, dementia, or any uncorrected visual disturbance (other than hemianopia).

There were 14 patients tested. Clinical and demographic data are summarised in table 1. Duration after stroke onset ranged between 2 to 12 weeks. Most (12) patients had significant left hemiparesis and substantial functional limitation. Five patients had hemianopic field defects to confrontation testing. Most patients had large lesions involving multiple right sided structures. Seven had frontal lesions, but only two were relatively isolated. Eleven had significant deep, lenticulostriate lesions, not surprising in a population in acute rehabilitation with hemiparesis, but only four were relatively isolated. Seven had posterior damage, but only one was isolated.

Thirteen right handed age and sex matched control participants were also included. The mean age of the control group was 68 years (SD 13.0; range 43 to 77). All control participants were without history of neurological or eye disease.

\section{Procedure}

The method was designed to attempt to replicate the findings of Marshall and Halligan. ${ }^{5}$ Participants were asked to bisect a total of 50, 7 inch $(17.8 \mathrm{~cm})$ horizontal lines that were drawn on different shaped pieces of paper. There were 10 trials each in which the lines were centred on rectangular sheets of white $9 \times 12$ inch paper, on square sheets of paper ( 12 inch sides), on circular sheets of paper (diameter 12 inches), and on isosceles triangular sheets of paper (axis 12 inches) -with the base of the triangle positioned perpendicular to the horizontal line target and the vertex pointed to the left or right (fig 1 ).

The 10, 7 inch horizontal lines centred within the rectangular sheets of paper were used as a baseline measure of bisection error. Based on the Halligan and Marshall report, ${ }^{4}$ this condition should have produced the most severe bisection error. It was reasoned that if a patient showed minimal error in this condition, there would likely not be enough "room for improvement" to evaluate the potential beneficial effect of the square and circle (and triangles). A mean deviation of $4 \mathrm{~mm}$ to the right was chosen as a minimum bisection error in order for patients to be administered the remaining context conditions. Four millimeters was chosen somewhat arbitrarily based on previous line bisection tasks in which we have shown that normal control participants rarely, if ever, produce deviations greater than $4 \mathrm{~mm}$ with a 7 inch line.

Each laminated stimulus sheet of paper was presented separately and always positioned on a tray top draped with a grey cloth in front of the subject so that the objective midpoint lay in the sagittal midplane of the subject's trunk. The order of the shaped pages was pseudorandomised so that no more than three trials of a particular shape were presented successively. Responses were made with a fine pen with erasable ink held in the right hand. Transection displacement from the true

Experiment 1: shaped paper
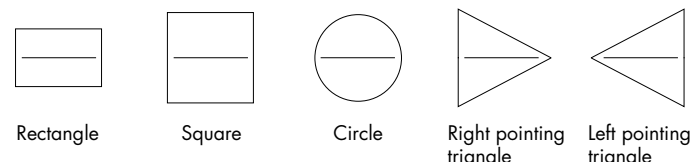

Rectangle
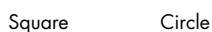

triangle

triangle

Experiment 2: outlined on paper

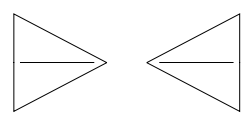

Right pointing Left pointing

triangle

Experiment 3: triangle components

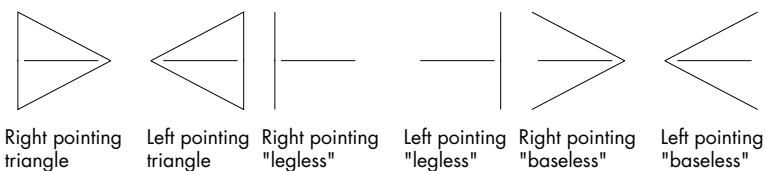

Figure 1 Example of the stimuli used in each of the experiments. In experiment 1, the stimulus paper on which the to be bisected line was drawn on was cut in the appropriate shapes, in experiments 2 and 3 , the triangular backgrounds were drawn on a piece of $18 \times 24$ inch piece of paper. 


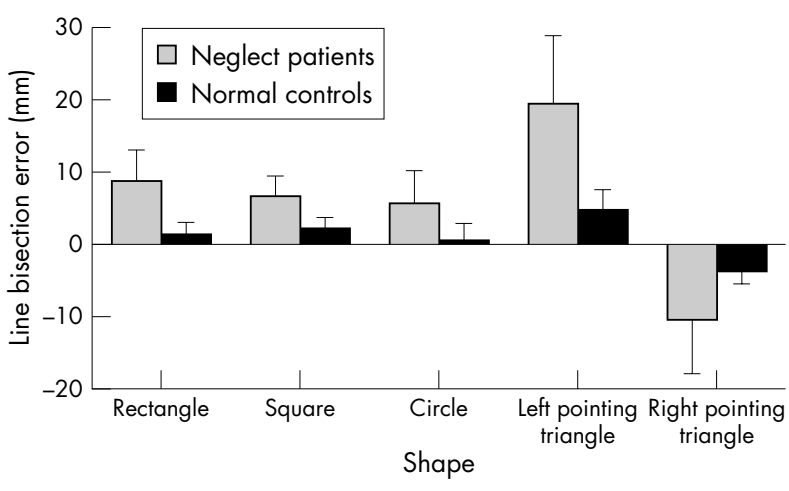

Figure 2 Mean bisection error (SD) for patients with neglect and controls as a function of shape in experiment 1 .

midpoint was measured to the closest millimeter and expressed as + for rightward and - for leftward bias.

\section{Results}

The mean deviation from the true midpoint is provided in figure 2 for patients with hemispatial neglect and controls. Analysis of variance (ANOVA) indicated main effects of group $(F(1,25)=38.44, \mathrm{p}<0.001)$, shape $(F(1,25)=38.44, \mathrm{p}<0.001)$, and a significant interaction between shape and group $(F(4,100)=19.41, \mathrm{p}<0.001)$. Post hoc comparisons showed that line bisection performance was not significantly influenced by page shape for rectangles, squares, or circles for either the control group or the patient group (all $\mathrm{p}$ values $>0.1$; fig 2), although there was an overall tendency for bisection bias to decrease from rectangles (mean rightward deviation, $M=5.06)$ to squares $(M=4.37)$, to circles $(M=3.03)$.

The effect of the triangular background did, however, significantly influence bisection performance compared with all other backgrounds, particularly so for the patients. Using the rectangular shape as a conservative baseline, with the triangle pointed left, patients' line transection moved significantly to the right, worsening hemispatial neglect $(p<0.0001)$. Conversely, with the triangle pointed to the right, line transection moved to the left of midline (crossing over) $(\mathrm{p}<0.001)$. This same effect was evidenced in the control subjects. In each subject the point of transection always shifted towards the base of the triangle. To determine if patients and controls differed in propensity for transaction displacement (TD), a corrected ratio was calculated: (triangle left TD minus rectangle TD) divided by (triangle right TD minus rectangle TD). The mean ratio for the patients (0.7) did not differ significantly from that of the controls $(0.9)(p>0.4)$, suggesting that the magnitude of the shift was similar for patients and controls once the baseline bisection bias was controlled.

Comparison of the patients with frontal cortical lesions, large anterior subcortical lesions, or both with those whose lesions were limited to or included significant posterior damage did not disclose any significant differences in transection performance $(p>0.3)$. The effect of the triangles was to shift line transection toward the triangle's base to a similar extent in all patients.

\section{Summary of experiment 1}

We did not confirm the effect of background shape on line bisection reported by Halligan and Marshall ${ }^{4}$ : a stepwise reduction in bisection error when the horizontal line was contained within the context of a rectangle to square to circle. The same general pattern emerged overall, but the differences failed to reach significance. However, looking at the data on a case by case basis, there were three patients who did show the stepwise reduction error found by Halligan and Marshall (patients 2, 3, and 8). ${ }^{4}$ Interestingly, although not statistically significant, their mean bisection error (10.87) was greater than the mean bisection error of the remaining 11 patients (8.19). This at least raises the possibility that our group did not show the stepwise reduction found by Halligan and Marshall ${ }^{4}$ because of an overall difference in severity between their patient (60 mm bisection deviation) and our group (mean deviation: $9.1 \mathrm{~mm}$ ). It is important to note, however, that there were patients in our study who showed an even greater mean deviation error than the three with the stepwise reduction. For example, patient 12 showed the greatest mean error with the rectangular background (17.9), but showed the least error on the square (6.0).

The isosceles triangular background shape had a potent effect on line bisection performance in patients with hemispatial neglect. Left pointing triangles (left apex/right base) caused a shift in transection to the right, exacerbating the pre-existing bisection error in the patients, and right pointing triangles (right apex/left base) caused a shift in transection to the left, ameliorating rightward error in the patients. The triangle effects were present in every patient and in every normal control, and the effects were of a similar magnitude between the groups when controlling for the overall extent of bisection bias. Sensitivity to background shape during horizontal line bisection task is normal in patients with hemispatial neglect.

\section{EXPERIMENTS 2 AND 3 \\ Subjects}

While testing the initial patients in experiment $\mathrm{l}$, we recognised that it would be necessary to deconstruct the triangles to establish the critical component in shift of a perceived midpoint. To accomplish this required using drawn triangles on standard paper so that either the apex legs or the base could be omitted. The last six patients (9-14) and eight controls were tested in experiments 2 and 3.

\section{Method, experiment 2}

Isosceles triangles were drawn on a laminated $18 \times 24$ inch sheet of white paper, and subjects were asked to bisect a 7 inch line centred in the triangle. There were 10 trials of left pointing triangles and 10 trials of right pointing triangles (fig 1).

\section{Results}

Transections from the triangular sheets of paper from experiment 1 were compared with the transections of the outlined triangles from experiment 2 for the subgroups of patients and controls who performed both. There were no significant differences in the magnitude between experiments 1 and 2, of the lateral shift of bisection for patients, either for triangles pointed right $(p>0.1)$ or for triangles pointed left $(p>0.3)$, or for controls $(p>0.05)$. The relative effects of triangular backgrounds seen in experiment 1 were replicated in experiment 2; the point of transection always shifted away from the vertex towards the base of the triangle.

\section{Summary, experiment 2}

The isosceles triangle background had an equally potent effect on perceived midpoint of a line whether the background was literally a triangular paper or simply a triangle drawn on conventional paper. In neither case was the shape of the paper or the drawn outline an explicit factor in the instructed task.

\section{Method, experiment 3}

The outlined triangle was deconstructed by removing either both "legs" of the apex or the "base" of the triangle. Again there were 10 trials each for the six possible outlined shapes (full outlined left or right, "legless" left or right, and "baseless" left or right (fig 1).

\section{Results}

Eliminating the apex "legs" of either triangle left only the vertical base as a possible lateral cue. Line bisection with only the 


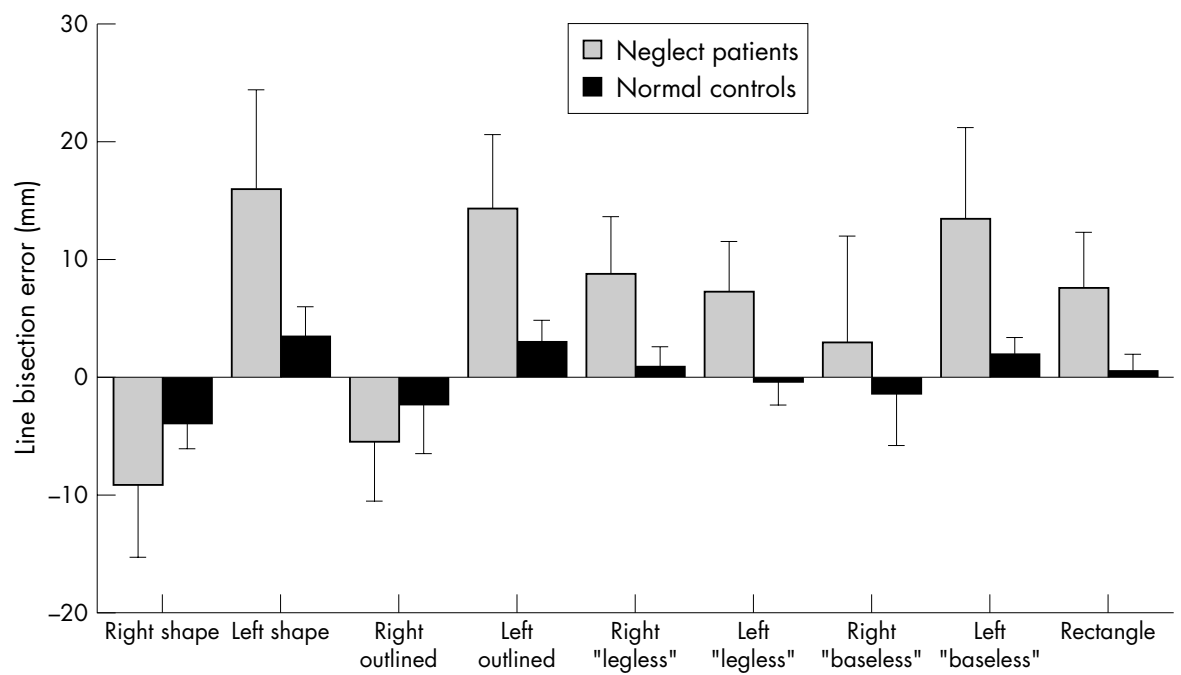

Figure 3 Mean bisection error (SD) for patients with neglect and controls for line bisections performed in the context of triangles across experiments 1, 2, and 3. Right and left shape and the baseline rectangle were from experiment 1, right and left outlined were from experiment 2 , and left and right "legless" and "baseless" were from experiment 3. Only those patients and controls that participated in all three experiments are represented.

vertical base remaining to either the left or the right was not significantly different from performance with the rectangular shaped background of experiment 1 in either patients or controls (all $\mathrm{p}$ values $>0.4$ ). Thus, shift of the perceived midpoint was lost without the vertex. By contrast, in triangles with the base eliminated, patient transections remained markedly shifted away from the apex comparable to the full triangle $(p>0.3)$ and significantly different from the baseline rectangle $(\mathrm{p}<0.001)$ (fig 3).

\section{Summary, experiment 3}

The vertex angle formed by the "legs" of the triangle is the crucial element that shifts perceived midpoint. With the "legs" of the triangle removed, leaving only the base adjacent to the horizontal line to be bisected, there was no difference in transection performance compared with our baseline condition (rectangle). The results do not support the hypothesis of Halligan and Marshall that a vertical component to the stimulus or the background could act as a cue and ameliorate neglect. ${ }^{4}$ However, the gap between the horizontal line and the unconnected vertical base was much greater in our experiment $(77 \mathrm{~mm})$ than in theirs $(4 \mathrm{~mm})$, perhaps explaining the lack of lateralising cueing in our experiment (fig 3).

\section{DISCUSSION}

Our experiments incorporated elements of background effects with elements of line illusions. Not surprisingly, either a background or feature context explanation can account for the potent effect of isosceles triangles.

The background account would be based on implicit-that is, preattentive-computation of the centre of mass of the triangle. Research on the accuracy of saccades to locate targets peripheral to fixation has demonstrated the importance of the centre of mass of a display. ${ }^{13}$ The role of the centre of mass calculation may serve only in the specific condition of shapes asymmetric across a vertical axis where the midpoint of a line centred within such a figure is different from its centre of mass. Thus, it is possible that when bisecting lines centred within triangles, patients with neglect shift lateral bias toward the centre of mass of the triangle. Patients with neglect differ from controls in the "centre point" from which bias shifts. It is unclear why patients with neglect show so much larger a bias shift than controls.
The stimulus feature account would be implicit-that is, preattentive sensitivity to geometric illusions. The Judd illusion (for example, $<-<$ or $>->$ ) is a shift of perceived midpoint. In both unilateral and bilateral fin Judd illusions, normal subjects, ${ }^{9}$ and patients with neglect ${ }^{78}$ make bisections that are displaced from the objective midline towards the outward projecting fin and away from the inward projecting fin. Stimulus features present in left hemispace that are neglected in explicit tasks, nevertheless influence bisection of the Judd illusion figures to the same extent as the explicitly perceived features present in the right hemispace. $^{8}$

The triangles in the current studies serve as a variation of the Judd fins. The triangular background is irrelevant to the line bisection task, but the "illusion" of line length centred within horizontally oriented isosceles triangles is distorted in a fashion similar to the perceived length of the Judd figure. The perceived midpoint is shifted to the opposite end (closer to the vertical base).

That the background for a stimulus or some biasing feature of the stimulus can affect visual neglect in patients with right brain damage has been demonstrated in many ways. The consistent induction of midpoint shift by the triangular background can be added to the list. Most explanations of these phenomena assert that they represent intact preattentive visual processing in the setting of impaired explicit visual attention. Work from our laboratory has specifically demonstrated that patients with hemispatial neglect can perform preattentive parallel searches normally but are impaired on attentive serial search. ${ }^{14}$ Even when no physical response into space is required, such as lexical decision, visual material that is neglected in explicit recognition tasks can still be processed for meaning. ${ }^{14} 15$

The two accounts presented above presume preattentive processing of information presented in the neglected hemispace. However, it should be considered whether the observed shifts in perceived midpoint could have been induced by the explicit perception of information available in the ipsilesional space. This possibility cannot be evaluated in the current study, due to the fact that on each trial, the background and line were presented bilaterally across the midline. Nevertheless, we can rule out this possibility based on another recently completed investigation in which the triangles were deconstructed into partial triangles. ${ }^{17}$ The triangles could have either originated from the vertex or base and, 
while presented in midline, could have been presented solely in left or right space, in the case of partial triangles $(50 \%$ or less). Consistent with the current study, the perceived midpoint always shifted toward the base of the triangle. Most relevant to the explicit account being considered here, there were no differential effects between patients with neglect and control participants of the partial triangles when they appeared to the left versus right of midline. There was an overall increase in bisection error to the right when the partial triangles appeared in the left versus the right, but to the same magnitude in both patients and control participants. In other words, the effect of a partial triangle appearing only in the non-neglected side of space was the same across groups as a partial triangle appearing only in the neglected side of space. These findings do not support an account of bisection shifts due only to explicit perception of the portion of triangles appearing in the right, ipsilesional side of space.

The current study, thus provides strong support for preserved preattentive visual processing, as the observed effects were seen in all 14 patients with neglect, mild or severe, with or without coexisting left hemianopia, and with all lesion locations-frontal, posterior, or both. It is certain that preattentive processes of various types are well preserved regardless of the neuroanatomical location of lesions that cause neglect. Halligan and Marshall ${ }^{4}$ have repeatedly shown preserved preattentive processes even in patients with extremely severe neglect and complete hemianopia due to extensive occipital infarctions in the right posterior cerebral artery territory. As even the presence of total left hemianopia and severe right occipital damage does not blunt these phenomena, the visual association cortex of either hemisphere must be capable of extracting and processing information without explicit attention or awareness. Although preserved preattentive processing of stimuli in left hemispace has been repeatedly demonstrated, a method to utilise these processes to ameliorate neglect remains elusive.

\section{ACKNOWLEDGEMENTS}

This work was supported by National Institute of Neurological Disease and Stroke Grant NS29342.

\section{Authors' affiliations}

M B Shulman, Department of Neurology, New York University Medical Center, New York, USA

M P Alexander, Department of Neurology, Beth Israel Deaconess

Medical Center, Harvard Medical School, Boston, MA, and Memory

Disorders Research Center, Boston University Medical School, Boston,

MA, USA

R McGlinchey-Berroth, W Milberg, Geriatric Research, Education and

Clinical Center, VA Boston Healthcare System and Department of

Psychiatry, Harvard Medical School, Boston, MA, USA

\section{REFERENCES}

1 Halligan PW, JC Marshall. How long is a piece of string? A study of line bisection in a case of visual neglect. Cortex 1988;24:321-8.

2 Chatteriee A. Cross-over, completion and confabulation in unilateral spatialone neglect. Brain 1995;118:455-65.

3 Anderson B. Pieces of the true crossover effect in neglect. Neurology 1997;49:809-12.

4 Halligan PW, Marshall JC. The bisection of horizontal and radial lines: A case study of normal controls and ten patients with left visuo-spatial neglect. Int J Neurosci 1993;70:149-69.

5 Marshall JC, Halligan PW. When right goes left: an investigation of line bisection in a case of visual neglect. Cortex 1989;25:503-15.

6 Marshall RS, Lazor RM, Krakamer JW, Sharma R, et al. Stimulus context in hemineglect. Brain 1998:121:2003-10.

7 Mattingley JB, Bradshaw JL, Bradshaw JA. The effects of unilateral visuospatial neglect on perception of Muller-Lyer illusory figures. Perception 1995;24:415-33.

8 Ro T, Rafal RD. Perception of geometric illusions in unilateral neglect. Neuropsychologia 1996;34:973-8.

9 Fleming J, Behrmann M. Visuospatial neglect in normal subjects: altered spatial representations induced by a perceptual illusion. Neuropsychologia 1998:36:469-75.

10 Shuren J, Wertman E, Heilman KM. The neglected page. Cortex 1994;30:171-5.

1 Halligan PW, Marshall JC. Grounding figural attention in left neglect. Neurocase 19́95;1:79-82.

12 Driver J. What can visual neglect and extinction reveal about the extent of "preattentive" processing. In: Logan GD, Coles MGH, Kramer AF, eds. Converging operations in the study of visual selective attention. Washington, DC: American Psychological Association, 1996:193-224.

13 Grabowecky M, Robertson LC, Treisman A. Preattentive processes guide visual search: Evidence from patients with unilateral visual neglect. $J$ Cogn Neurosci 1993;5:288-302.

14 Esterman M, McGlinchey-Berroth R, Milberg W. Preattentive and attentive visual search in individuals with hemispatial neglect. Neuropsychology 2000;14:599-611

15 McGlinchey-Berroth R, Milberg WP, Verfaillie M, et al. Semantic processing and orthographic specificity in hemispatial neglect. J Cogn Neurosci 1996:8:291-304.

16 McGlinchey-Berroth R, Milberg WP, Verfaillie M, et al. Semantic processing in the neglected visual field: Evidence from a lexical decision task. Cogn Neuropsychol 1993;10:79-108

17 Esterman M, McGlinchey-Berroth R, Alexander MP, et al. Triangular backgrounds shift the bias of line bisection performance in hemispatial neglect: The critical point. J Int Neuropsychol Soc (in press). 